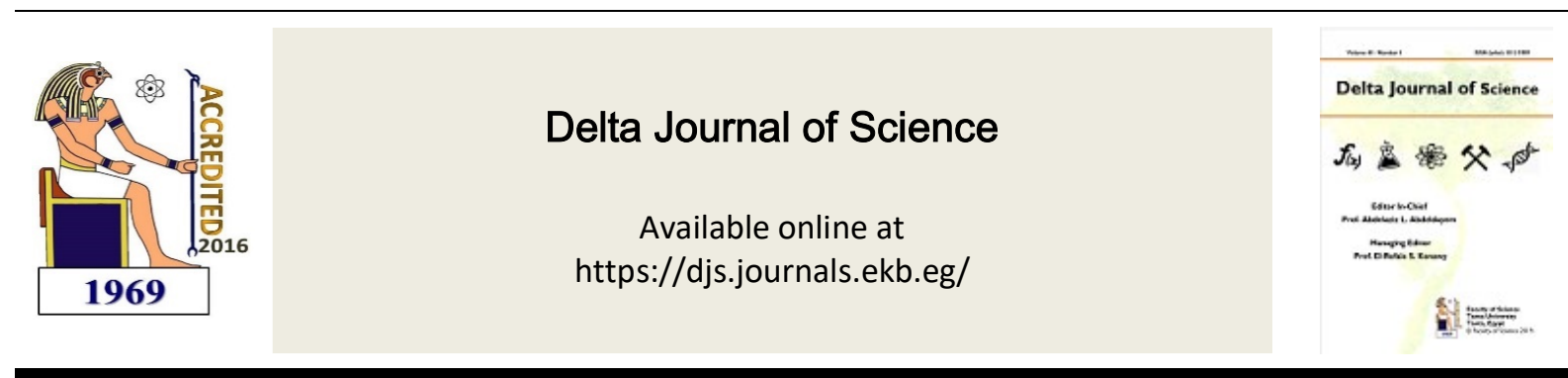

Research Article

MATHEMATICS

\title{
Perfect folding of graphs
}

E. M. El-Kholy and H. Ahmed

Department of Mathematics, Faculty of Science, Tanta University, Tanta, Egypt. Department of Mathematics, Faculty of Shoubra Engineering, BanhaUniversity ,Egypt.

\begin{tabular}{|c|c|}
\hline KEY WORDS & ABSTRACT \\
\hline $\begin{array}{l}\text { Clique } \\
\text { number, } \\
\text { chromatic } \\
\text { number, } \\
\text { perfect } \\
\text { graphs, graph } \\
\text { folding }\end{array}$ & $\begin{array}{l}\text { In this paper we introduced the definition of perfect folding } \\
\text { of graphs and we proved that cycle graphs of even number of } \\
\text { edges can be perfectly folded while that of odd number of } \\
\text { edges can be perfectly folded to } C_{3} \text {. Also we proved that } \\
\text { wheel graphs of odd number of vertices can be perfectly } \\
\text { folded to } C_{3} \text {. Finally we proved that if } G \text { is a graph of } n \text { vertic- } \\
\text { es such that } 2<\text { clique number =chromatic number } k<n \text {, } \\
\text { then the graph can be perfectly folded to a clique of order } k \text {. }\end{array}$ \\
\hline
\end{tabular}

\section{Introduction}

Let $G=(V, E)$ be a graph, where $V$ is the set of its vertices and $E$ is the set of its edges. Two distinct vertices $u, v \in V$ are called independent if $\{u, v\}$ is not an edge in $G$. Two vertices $u, v$ are called neighbors (adjacent) if $\{u, v\}$ is an edge in $G$. The degree (valency) of a vertex is the number of edges with the vertex as an end point. A graph with no loops or multiple edges is called a simple graph . A graph is said to be connected if every pair of vertices has a path connecting them otherwise the graph is disconnectted. A graph $H=\left(V, E^{\prime}\right)$ is called induced subgraph of $G=(V, E)$ if $V^{\prime} \leq V$ and $\{u, v\}$ is an edge in $H$ wherever $u$ and $v$ are distinct vertices in $\mathrm{V}^{\prime}$ and $\{u, v\}$ is an edge in $G, H$ is called proper if $H \neq G$. A cycle graph is a graph that consists of a single cycle, or in other words, some number of distinct vertices connected in a closed chain. The cycle graph with $n$ vertices is denoted by $C_{n}$. The number 
of vertices in $C_{n}$ equals the number of edges, and every vertex has degree 2 . The wheel graph $W_{n}$ or $n$-wheel is a graph that contains a cycle of order $n-1$, and for which every graph vertex in the cycle is connected to one other graph vertex which is called the hub. A bipartite graph is a graph whose vertex set can be split into two sets $A$ and $B$ in such a way that each edge of the graph joins a vertex in $A$ to a vertex in $B$. A vertex coloring of a graph $G=(V, E)$ is a way of coloring the vertices of the graph such that no two adjacent vertices share the same color. A clique of a graph $G$ is a maximal complete subgraph. In this case each pair of vertices of the clique are adjacent. The clique number $W(G)$ of a graph is the number of graph vertices in the largest clique of $G,[8]$. The clique number of a cycle graph $C_{n}, n$ odd is 3 and 2 otherwise. For a wheel graph $W_{n}$, $n$ is even the clique number is 4 and is 3 otherwise. The chromatic number of a graph $G$ is the smallest number of colors needed to color the vertices of a graph $\mathrm{G}$ so that no two adjacent vertices share the same color, and is often denoted by $\chi(\mathrm{G})$. A graph $G$ is called perfect if for every induced subgraph $H$ of $G, \chi(H)=W(H)$. Note that if $\mathrm{G}$ is a perfect graph, then every induced subgraph of $\mathrm{G}$ is also perfect,[2].

\section{(2)Perfect folding}

\section{Definition (2-1)}

Let $G_{1}$ and $G_{2}$ be two simple graphs and $f: G_{1} \rightarrow G_{2}$ be continuous map. Then $f$ is called a graph map, if (i) For each vertex $v \in V\left(G_{l}\right), f(v)$ is a vertex in $V\left(G_{2}\right)$. (ii) For each edge $e \in E\left(G_{l}\right), \operatorname{dim}(f(e)) \leq$ $\operatorname{dim}(e),[3]$.

\section{Definition (2-2)}

A graph map $f: G_{1} \rightarrow G_{2}$ is called a graph folding if and only if $f$ maps vertices to vertices and edges to edges, i.e., if

(i) For each vertex $v \in V\left(G_{1}\right), f(v)$ is a vertex in $V\left(G_{2}\right)$.

(ii) For each edge $e \in E\left(G_{1}\right), f(e)$ is an edge in $E\left(G_{2}\right)$, [4] .

Note that if the vertices of an edge $e=(u, v) \in E\left(G_{1}\right)$ are mapped to the same vertex, then the edge e will collapse to this vertex and hence we cannot get a graph folding. In other words, any graph folding cannot maps edges to loops but it may maps loops, if there is any, to loops.

\section{Definition (2-3)}

Let $G$ and $H$ be simple connected graphs. We call a graph folding $f: G \rightarrow H$ perfect folding if its image $f(G)$ is a perfect subgraph of $H$.

In general the image of a graph folding $f: G \rightarrow H$ is not a perfect graph e.g., if $G_{l}$ is the imperfect graph shown in Fig.(1-a), where $V\left(G_{1}\right)=\left\{v_{1}, v_{2}, v_{3}, v_{4}, v_{5}\right.$, $\left.v_{6}, v_{7}\right\}$ and $E\left(G_{1}\right)=\left\{e_{1}, e_{2}, e_{3}, e_{4}, e_{5}, e_{6}\right.$, $\left.e_{7}\right\}$. Then the graph folding $f: G_{1} \rightarrow G_{1}$ defined by $f\left\{v_{6}, v_{7}\right\}=\left\{v_{5}, v_{4}\right\}$ and $f\left\{e_{6}, e_{7}\right\}=\left\{e_{2}, e_{4}\right\}$ is not a perfect folding. While if we consider the imperfect graph $G_{2}$ shown in Fig.(1-b), where $V\left(G_{2}\right)=\left\{u_{1}, \ldots, u_{7}\right\}$ and $E\left(G_{2}\right)=\left\{e_{1}, \ldots\right.$, $\left.e_{7}\right\}$. Then the graph folding $g: G_{2} \rightarrow G_{2}$ defined by $g\left\{u_{1}, u_{4}\right\}=\left\{u_{6}, u_{6}\right\}$ and $g\left\{e_{4}\right.$, $\left.e_{7}\right\}=\left\{e_{5}, e_{6}\right\}$ is a perfect folding. The omitted vertices and edges in this 
example and through the paper will be mapped to themselves.
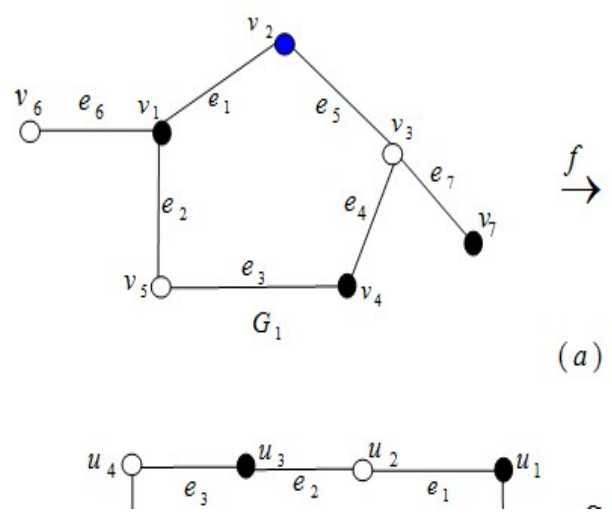

Fig.(1)

\section{Theorem (2-1)}

Let $G$ be a simple connected graph such that the number of $E(G) \geq 2$. If the chromatic number $\chi(G)$ is equal to two, then $G$ can be perfectly folded.

\section{Proof}

From [5], any simple connected graph $\mathrm{G}$ such that $E(G) \geq 2$ and $\chi(G)=2$ can be folded to an edge. In this case $\chi(f(G))=$ $W(f(G))=2$, and thus the graph $G$ can be perfectly folded to an edge.

\section{Example (2-4)}

The cubic graph $\mathrm{G}$ with $\chi(G)=W(G)$ $=2$, shown in Fig. (2) can be folded to an edge by the graph folding $f\left(v_{1}, \ldots\right.$, $\left.v_{8}\right)=\left(v_{1}, v_{2}, v_{1}, v_{2}, v_{1}, v_{2}, v_{1}, v_{2}\right)$. This folding can be done by the composition of a sequence of foldings $f_{1}, f_{2}, f_{3}$ and $f_{4}$, see Fig.(2) . And hence the graph folding is a perfect.
Fig.(2)

\section{Lemma (2-2)}

Any folding of a bipartite graph (complete) is a perfect folding. Proof

This follows from the fact that the chromatic number of a bipartite graph is equal to two, and thus it can be perfectly folded.

\section{Example (2-5)}

Consider the bipartite graph $\mathrm{G}$ shown in Fig.(3). A graph folding $f: G>G$ defined by $f\left\{v_{1}, v_{3}\right\}=\left\{v_{2}\right\}$ and $f\left\{e_{1}, e_{4}\right\}=$ $\left\{e_{2}, e_{3}\right\}$ is a perfect folding.

\section{(3) Perfect folding of cycle graphs}

The chromatic number of a cycle graph $C_{n}, n>2$ where $n$ is odd is 3 while that for $n$ even is $2,[1]$.

\section{Theorem (3-1)}

Any folding of a cycle graph $C_{n}$ of an even number of edges is a perfect folding.

\section{Proof}

This follows from the fact that $\chi\left(C_{n}\right)$, $n$ is an even number is equal to two. Thus $C_{n}$ can be perfectly folded.

\section{Example (3-1)}

Consider the cycle graph $C_{4}$ where

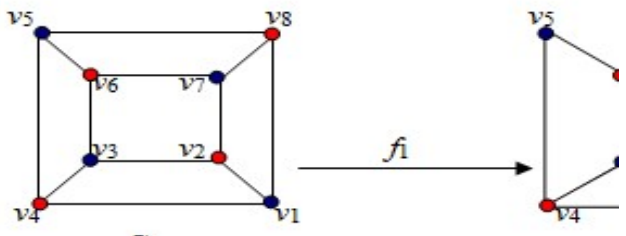
$\chi\left(C_{4}\right)=W\left(C_{4}\right)=2$, the graph folding 
$f: C_{4} \rightarrow C_{4}$ defined by $f\left\{v_{1}, v_{4}\right\}=\left\{v_{3}, v_{2}\right\}$ and $f\left\{e_{i}\right\}=\left\{e_{3}\right\}, i=1,2,4$ is a perfect folding, see Fig.(4).

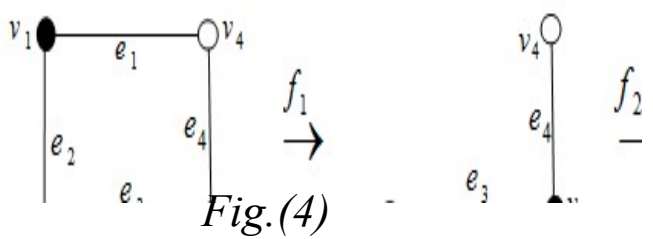

It should be noted that the cycle graph $\mathrm{C}_{3}$ cannot be folded, [4].

\section{Theorem (3-2)}

Let $G=C_{n}, n>3$ be a cycle graph of an odd number of edges (vertices). Then $G$ can be perfectly folded to $C_{3}$.

\section{Proof}

Since $G=C_{n}$ has an odd number of edges (vertices). Thus the graph $C_{n}$ has three color classes, say $V_{1}, V_{2}$ and $V_{3}$. We can color the vertices of $C_{n}$ alternatively with the two colors of $V_{l}$ and $V_{2}$ except the last two edges one will join a vertex colored by the color of $V_{2}$ and a vertex colored by the color of $V_{3}$ and the other edge will join a vertex colored by the color of $V_{3}$ and a vertex colored by the color of $V_{l}$. Thus the number of vertices of color class $V_{1}=$ the number of vertices color class $V_{2}=(n-1) / 2$, but $V_{3}$ has only one vertex $w$. We can define a graph folding $f: C_{n}$ $\rightarrow C_{n}, n$ is odd, by mapping vertices of $V_{l}$ to a vertex of $V_{1}$, say $u$, and mapping the vertices of $V_{2}$ to a vertex of $V_{2}$, say $v$, finally mapping $w$ into itself. Thus we have three vertices $u, v, w$ and hence three edges in the image i.e., we have $C_{3}$. But $\chi\left(C_{3}\right)=W\left(C_{3}\right)=3$, i.e., the graph folding $f$ is perfect.

\section{Example (3-2)}

Let $G=C_{5}$ and $h: G \rightarrow G$ be the graph folding defined by $h\left\{v_{5}, v_{4}\right\}=\left\{v_{3}, v_{1}\right\}$ and $h\left\{e_{i}\right\}=\left\{e_{2}\right\}, i=3,4$ is a perfect folding, see Fig.(5). This can be done by the composition of the two graph foldings $h_{1}: C_{5} \rightarrow C_{5}$ defined by $h_{1}\left\{v_{5}\right\}=\left\{v_{3}\right\}$, $h_{1}\left\{e_{3}\right\}=\left\{e_{4}\right\}$ and $h_{2}: h_{1}\left(C_{5}\right) \rightarrow h_{1}\left(C_{5}\right)$ defined by $h_{2}\left\{v_{4}\right\}=\left\{v_{1}\right\}, h_{2}\left\{e_{4}\right\}=\left\{e_{2}\right\}$.

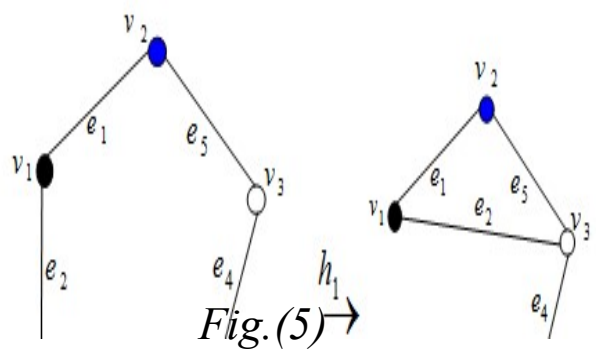

(4) Perfect folding of wheel graphs

The chromatic number of a wheel graph $W_{n}$ is 3 ; if $n$ is odd ; and 4 ; if $n$ is even, [1].

\section{Theorem (4-1)}

Any wheel graph $W_{n}$ of an odd number of vertices can be perfectly folded to $C_{3}$.

Proof:

A wheel graph $W_{n}$ of order $n, n$ is an odd number, is a graph that contains a cycle of even order $n-1$, and each vertex in the cycle is connected to the hub. In this case the chromatic number $\chi\left(W_{n}\right)=3$, thus the graph $W_{n}$ can be colored by using three colors $A, B$ and $C$. One color for the hub, say $A$, and the vertices of the even cycle $C_{n-1}$ can be colored alternatively with two colors $B$ and $C$, i.e., if the set of vertices of the cycle $C_{n-1}$ is $V\left(C_{n-1}\right)=\left\{v_{1}, v_{2}, \ldots, v_{n-1}\right\}$, then the colors $B$ and $C$ have the 
following vertices, $B=\left\{v_{1}, v_{3}, \ldots, v_{n-2}\right\}$ and $C=\left\{v_{2}, v_{4}, \ldots, v_{n-l}\right\}$. Now we can define a graph folding by mapping the vertices of $B$ to a vertex of $B$, the vertices of $C$ to a vertex of $C$ and the hub onto itself. The image of this map will contains three vertices, three edges and thus we have $C_{3}$, i.e., the graph folding is perfect.

\section{Example (4-1)}

Consider the wheel graph $W_{7}$ and the graph folding $f: W_{7} \rightarrow W_{7}$ defined by $f\left\{v_{i}\right\}=\left\{v_{1}\right\}, i=3,5, f\left\{v_{j}\right\}=\left\{v_{2}\right\}, \mathrm{j}=4,6$ and $f\left\{e_{k}\right\}=\left\{e_{1}, e_{1}, e_{1}, e_{1}, e_{1}, e_{1}, e_{7}, e_{8}, e_{7}, e_{8}\right.$, $\left.e_{7}, e_{8}\right\}, k=1, \ldots, 12$. This graph folding is perfect, see Fig.(6).

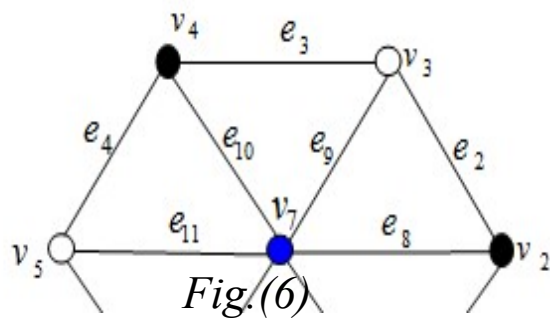

It should be noted that the wheel graph of an even number of vertices cannot be folded, [4], and hence cannot be perfectly folded.

\section{(5) The clique number and perfect folding}

The chromatic number of any graph is equal to or greater than its clique number, i.e., $\chi(G)>W(G)$. For connected graphs $2 \leq W(G) \leq \chi(G) \leq n$, where $n$ is the number of vertices of the graph $G$, [7].

\section{Theorem (5-1)}

Let $G$ be a simple connected graph, if the clique number $W(G)$ equal to the chromatic number $\chi(G)$ equal to 2 and
$E(G) \geq 2$, then the graph $G$ can be perfectly folded.

\section{Proof}

It is immediately follows from

Theorem (2-4) and since $\chi(G)=2$, then $G$ can be perfectly folded.

\section{Example (5-1)}

Consider the cycle graph $C_{6}$ shown in Fig.(7). A graph folding $f: C_{6} \rightarrow C_{6}$ defined by $f\left\{v_{2}, v_{3}, v_{4}, v_{5}\right\}=$ $\left\{v_{6}, v_{1}, v_{6}, v_{1}\right\}$ and $f\left\{e_{i}\right\}=\left\{e_{6}\right\}, i=1, \ldots, 5$ is a perfect folding.

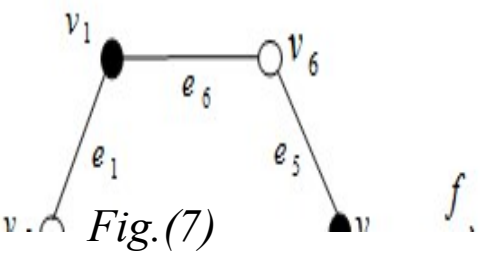

\section{Theorem (5-2)}

Let $G$ be a simple connected graph such that $n o . V(G)=n$. If $2<W(G)=$ $\chi(G)=k<n$, then the graph can be perfectly folded to a clique of order $k$.

\section{Proof}

Let $W(G)=\chi(G)=k$, then we have a maximal complete subgraph of $k$ vertices. This complete graph cannot be folded, [3]. These vertices must be colored by different colors $A_{1}, A_{2}, \ldots, A_{k}$. Now the other $(n-k)$ vertices of $G$, will be colored by the colors $A_{1}, \ldots, A_{m}, m \leq k$ in such a way that any edge will joins two vertices of different colors. So we can define a seque- nce of graph folding $f_{i}: G \rightarrow G_{i}$, where $G_{i}=f_{i}\left(G_{i-1}\right), i=$ $1, \ldots, m, G_{0}=G$, by mapping the $(n-k)$ vertices to other vertices but of the same color, until we get the $k$-clique 
which cannot be folded any more. And hence $W\left(f_{i}\left(G_{i}\right)\right)=\chi\left(f_{i}\left(G_{i}\right)\right)=k$, i.e., the graph folding is a perfect.

\section{Example (5-2)}

Consider the house graph $G$ with 5 vertices and 6 edges shown in Fig.(8), where $2<W(G)=\chi(G)=3<n=5$. This graph can be folded to a triangle by the graph folding $f: G \rightarrow G$ defined by $f\left\{v_{4}, v_{5}\right\}=\left\{v_{2}, v_{3}\right\}$ which is a perfect folding.

\section{References}

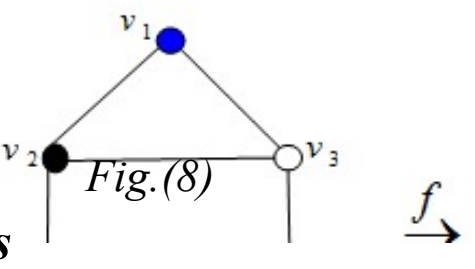

[1]Bollobas, B. and West, D. B. " A note on generalized chromatic number and generalized girth" Disc. Math.213, 2934 , (2000).

[2] Balakrishnan, R. and Ranganathan, $K$. "A text book of graph theory" Springer, New York, (1991).

[3] Erdos, P. "Graph theory and probability" Canad. J. Math. 11, 34-38, (1959).

[4] El-Kholy, E.M. and Al-Esawy, A. "Graph folding of some special graphs" J. Math. \& Statistics 1(1), 66-70, (2005). [5] EL-Kholy, E. and EL-Sharkawe, $N$. : The chromatic number and graph folding" European J. Scientific Research, Vol.120, No.1, 138-144, (2014).

[6] Golumbic, M. C. " Algorithmic graph theory and perfect graphs" Annals of Discr. Math.7 , (2004). [7] Lovasz, L. "Normal hypergraphs and the weak perfect graph cojecture" Annals of Discr. Math.21,29-42, (1984).
[8]Lowell, W. and Robin, J. "Selected topics in graph theory2" Academic Press Inc. (London) LTD , (1983). 
